\section{Surgically induced miosis during phacoemulsification in patients with diabetes mellitus}

SA Mirza', A Alexandridou', T Marshall ${ }^{2}$ and P Stavrou'
${ }^{1}$ Birmingham and Midland Eye Centre, City Hospital Birmingham, UK

${ }^{2}$ Department of Public Health and Epidemiology University of Birmingham Birmingham, UK

Correspondence:

P Stavrou

Birmingham and Midland Eye Centre

City Hospital NHS Trust

Dudley Road

Birmingham B18 7QU, UK

Tel: +4401215076854

Fax +44 01215076853

E-mail: panagiotastavrou@

hotmail.com

Received: 6 December 2001 Accepted: 17 April 2002

\begin{abstract}
Purpose To assess the incidence of surgically induced miosis during phacoemulsification in diabetic patients.

Methods A total of 76 patients with diabetes mellitus were compared to 76 age- and race-matched controls. A combination of cyclopentolate $1 \%$, phenylephrine $2.5 \%$ and diclofenac sodium $0.1 \%$ was applied topically 60, 45 and $30 \mathrm{~min}$ before surgery. Adrenaline mixed with buffered saline solution was used for irrigation during surgery. The procedure included phacoemulsification and implantation into the bag of a foldable acrylic implant. Measurements of the horizontal pupillary diameter were taken at three stages: before corneal incision, after phacoemulsification, and at the end of surgery. The duration of phacoemulsification was also recorded.

Results Surgically induced miosis or dilation of the pupil was defined as constriction or dilation noted at any interval during surgery. The pairs of diabetic-control were grouped into three groups: those in which constriction was noted, those in which dilation was noted, and those in which there was no change in pupil size during the procedure. Surgically induced miosis was noted more often in the diabetics (McNemar's test, $\chi^{2}, P=0.016$ ). The mean pupil size at the beginning of surgery was $7.38( \pm 0.95) \mathrm{mm}$ in the diabetics as compared to $7.65( \pm 0.89) \mathrm{mm}$ in the control group. No statistically significant difference was noted between the two groups (paired $t$ test, $P=0.07)$. The mean $( \pm S D)$ duration of phacoemulsification in the diabetic group was $2.31( \pm 1) \mathrm{min}$ as compared to $2.05( \pm 0.82)$ $\min$ in the control group. No statistically significant difference was found between the two groups (paired $t$-test, $P=0.08$ ).
\end{abstract}

Conclusion Surgically induced miosis occurred more often in the diabetics. Therefore, it is advisable that phacoemulsification in this group of patients is undertaken by an experienced surgeon. Eye (2003) 17, 194-199. doi:10.1038/ sj/eye/6700268

Keywords: diabetes mellitus; phacoemulsification; cataract; pupil; diclofenac sodium

Introduction

Cataract is one of the most important changes observed in the lens in diabetes and a major contributor to diabetic blindness. The prevalence of cataract is increased in diabetics, especially in women. ${ }^{1,2}$ Previous studies have shown an association between the development of cataract and diabetes duration, retinopathy and life expectancy. ${ }^{1-4}$ The frequency of cataract extraction is also greater in diabetics than nondiabetics. ${ }^{5}$ Other lens changes in diabetes include increased lens thickness, which is mainly because of cortical thickening, and biometric changes such as steepening of the front and back curvature of the lens and shallowing of the anterior chamber. ${ }^{6}$

The size of the pupil is under the control of the parasympathetic system, which innervates the sphincter pupillae muscle, and the sympathetic system, which innervates the dilator pupillae muscle. In diabetics, the resting pupil is smaller than in healthy subjects, characterised by failure to dilate in the darkness. ${ }^{7}$ The small pupil in diabetic patients is supersensitive to phenylephrine. ${ }^{7}$ The pupil of the diabetics in the dark is inversely related to the diabetes duration and level of blood glucose throughout several years; those with 
proliferative retinopathy have smaller size pupil than those without. ${ }^{8}$ In continuous light, the long-term diabetics have a smaller pupil size than the controls. ${ }^{8}$ The failure of the diabetic pupil to dilate in the dark is because of neuropathy of the sympathetic innervation. ${ }^{7}$ Degenerative changes affecting predominantly the dilator muscle have also been reported in diabetics. ${ }^{9}$

The commonly used anticholinergic agents such as homatropine, cyclopentolate, and tropicamide act by paralysing the parasympathetic constrictor drive, allowing the sympathetic input to the dilator to dominate. The loss of sympathetic tone in patients with diabetic neuropathy explains why the addition of a directly acting sympathomimetic, to which the diabetic pupil is hypersensitive, produces adequate mydriasis in diabetics, comparable to that seen in healthy subjects. ${ }^{10}$ However, in the same study, it was noted that the pupils of diabetic patients whose eyes had previously received laser treatment dilated less than those from untreated eyes. The authors suggested that it was not clear if the laser treatment itself or the more advanced stage of diabetic retinopathy was responsible for the pupil being more resistant to mydriatics. ${ }^{10}$

Cataract surgery is technically easier to perform when a well-dilated pupil can be maintained throughout the procedure. The aim of this study was to assess surgically induced miosis in a group of diabetics undergoing phacoemulsification as compared to an age- and racematched control group. Both diabetic and control patients were treated preoperatively with a combination of adrenergic, anticholinergic, and a topical prostaglandin (PG) synthesis inhibitor (Diclofenac Sodium, Voltarol, Novartis Ophthalmics). We also compared the size of the pupil at various times during the procedure as well as the duration of the phacoemulsification in the two groups.

\section{Patients and methods}

Patients with diabetes mellitus undergoing cataract extraction by phacoemulsification by one surgeon (PS) were included in the study. Data were collected prospectively from patients operated on between March 2000 and November 2001. Patients were classified as diabetics on the basis of history. We included patients on diet alone, oral hypoglycaemics, and/or insulin. In cases of bilateral cataract surgery, only the first eye was included. The diabetics were matched for age and race to healthy controls operated on during the same period by the same surgeon for senile cataract. The controls were identified from the surgeon's personal database, which includes all patients undergoing cataract surgery. All healthy individuals had urine specimen analysis to exclude undiagnosed diabetes mellitus. Exclusion criteria were other ocular pathology such as uveitis, glaucoma, pseudoexfoliation, rubeosis iridis, previous ocular surgery or trauma to the operated eye, concurrent medication with oral PG synthesis inhibitor for systemic illness, known hypersensitivity to diclofenac sodium or other PG synthesis inhibitor, and the use of any topical ophthalmic medication, other than lubricants, in the preceding 2 weeks.

All patients received a standard preoperative regimen of one drop of cyclopentolate $1.0 \%$, one drop of phenylephrine $2.5 \%$, and one drop of diclofenac $0.1 \%$ given at 60, 45, and $30 \mathrm{~min}$ prior to surgery. Anaesthesia was given by peribulbar injection via the medial canthus using prilocaine hydrochloride $4 \%$ and lignocaine $2 \%$ in equal proportions combined with hyaluronidase.

Phacoemulsification was performed through a standard superior clear corneal incision using the 'divide-andconquer' technique and manual aspiration-irrigation with the Simcoe cannula. Adrenaline, $0.5 \mathrm{ml}$ of $1: 1000$, added to $500 \mathrm{ml}$ of balanced salt solution was used for irrigation during the procedure. A foldable acrylic intraocular lens was implanted in the capsular bag in all cases.

Horizontal pupillary diameter measurements were obtained by the surgeon, to the nearest $0.25 \mathrm{~mm}$, using Vernier callipers before corneal incision was made (first measurement), at the end of phacoemulsification (second measurement) and at the end of surgery (third measurement). All measurements were taken without viscoelastic being present and with the anterior chamber filled with 'balanced salt solution'. The duration of phacoemulsification was recorded for each case as the 'elapsed time' in minutes displayed on the phacoemulsifying machine at the end of the procedure. Phacoemulsification was performed using one machine and the same parameters for all patients and all grades of cataract so that the duration of phacoemulsification could be comparable. Patients with intraoperative complications such as vitreous loss or those in whom phacoemulsification had to be converted to extracapsular technique were excluded from the study.

Statistical analysis of the size of the pupil at the beginning of surgery and of the duration of phacoemulsification in the diabetics compared with the controls was made by using paired $t$-test. Surgically induced miosis or dilation of the pupil was defined as constriction or dilation noted at any interval, that is between first and second measurements or between second and third measurements. The pairs of diabeticcontrol were grouped into three groups: those in which constriction was noted, those in which dilation was noted and those in which there was no change in pupil size during the procedure. Statistical comparison of the intraoperative constriction or dilation was made by 
applying McNemar's $\chi^{2}$ test to the number of pairs of diabetic-control showing intraoperative constriction or dilation.

\section{Results}

In all, 76 eyes of 76 diabetic patients were included in the study. The control group consisted of 76 eyes of 76 healthy patients matched for age and race to the diabetic group. The mean $( \pm \mathrm{SD})$ age in the diabetic group was $70.3( \pm 8.8)$ years as compared to $70.8( \pm 9.1)$ years in the control group (Table 1). The male/female ratio was $44 / 32$ in the diabetic group and 35/41 in the control group. In both groups there were 38 Asians, 25 Caucasians, 10 Afro-Caribbeans, and three Chinese. In the diabetic group, the median $( \pm$ SEM) duration of diabetes prior to cataract surgery was $8( \pm 1.2)$ years [range $1-50$ years]. In all, 46 patients had no clinical evidence of diabetic retinopathy, 22 patients had background diabetic retinopathy, five had undergone laser for diabetic maculopathy, and the remaining three patients had undergone laser for proliferative diabetic retinopathy. Patients who had required laser treatment for maculopathy or proliferative retinopathy had been stable for several months prior to undergoing cataract surgery. White cataract, defined as a totally opacified lens precluding visualisation of the red reflex, was noted in nine patients with diabetes and in 11 controls. In diabetic patients with white cataract, the stage of diabetic retinopathy was assessed at the first postoperative day.

The mean $( \pm S D)$ pupil size before corneal incision (first measurement) in the diabetic group was 7.38 ( \pm 0.95$) \mathrm{mm}$ [range $5-9.5 \mathrm{~mm}$ ] as compared to 7.65 $( \pm 0.89) \mathrm{mm}$ [range $5-9 \mathrm{~mm}$ ] in the control group. No

Table 1 Patient demographics

\begin{tabular}{lcc}
\hline & Diabetics & Controls \\
\hline No. of patients & 76 & 76 \\
Mean $( \pm$ SD) age (years) & $70.3( \pm 8.8)$ & $70.8( \pm 9.1)$ \\
$\quad$ Range & $47-78$ & $47-80$ \\
Race & & \\
$\quad$ Asian & 38 & 38 \\
Caucasian & 25 & 25 \\
$\quad$ Afro-Caribbean & 10 & 10 \\
Chinese & 3 & 3 \\
Sex & & \\
$\quad$ Male & 44 & 35 \\
$\quad$ Female & 32 & - \\
Median $( \pm$ SEM) duration & & \\
of diabetes (years) & $8( \pm 1.2)$ & - \\
$\quad$ Range & $1-50$ & \\
Diabetes mellitus & & \\
$\quad$ Type 1 & 2 & \\
Type 2 & 74 & \\
\hline
\end{tabular}

Table 2 Change of pupil during phacoemulsification in pairs of diabetic-control

\begin{tabular}{lccc}
\hline & \multicolumn{2}{c}{ Diabetics } \\
\cline { 3 - 4 } & Constriction & $\begin{array}{c}\text { No change/ } \\
\text { dilation }\end{array}$ \\
\hline Controls & $\begin{array}{c}\text { Constriction } \\
\text { No change/dilation }\end{array}$ & 12 & 9 \\
& 24 & 31 \\
\hline
\end{tabular}

statistically significant difference was seen in the pupil size before corneal incision between the two groups $(P=0.07$, paired $t$-test). The mean $( \pm \mathrm{SD})$ pupil size at the end of phacoemulsification (second measurement) in the diabetic group was $7.11( \pm 1.2) \mathrm{mm}$ [range $4-9.5 \mathrm{~mm}$ ] as compared to $7.63( \pm 1) \mathrm{mm}$ [range $4-9 \mathrm{~mm}$ ] in the control group. The difference was statistically significant $(P=0.005$, paired $t$-test $)$. Furthermore, the mean $( \pm S D)$ pupil size at the end of surgery (third measurement) in the diabetic group was $7.13( \pm 1.3) \mathrm{mm}$ [range $4-9 \mathrm{~mm}$ ] as compared to $7.65( \pm 1.1) \mathrm{mm}$ [range $4-9 \mathrm{~mm}$ ] in the control group. This difference was also statistically significant $(P=0.01$, paired $t$-test). The mean $( \pm \mathrm{SD})$ duration of phacoemulsification in the diabetic group was $2.31( \pm 1) \mathrm{min}$ [range $1.09-4.49 \mathrm{~min}$ ] as compared to $2.05( \pm 0.82) \mathrm{min}$ [range 1.02-4.7 min] in the control group. No statistically significant difference was seen in the duration of phacoemulsification between the two groups ( $P=0.08$, paired $t$-test).

Table 2 shows the number of pairs of diabetic-control according to intraoperative constriction/dilation or no change in pupil size. As it was considered that intraoperative constriction is of higher clinical significance than intraoperative dilation, pairs showing intraoperative dilation or no change in pupil size were grouped together. In Table 2, numbers 12 and 31 are uninformative pairs, as they do not contribute to the discrimination between diabetics and controls with respect to pupil size during surgery. However, in 24 pairs the diabetic showed intraoperative constriction while the control did not. Furthermore, in nine pairs the control showed intraoperative constriction while the diabetic did not. Applying McNemar's test to the pairs in which only one of the two individuals comprising a pair showed intraoperative constriction, that is the 24 pairs were compared to the nine pairs, demonstrated that intraoperative constriction occurred more often in the diabetics $\left(\chi^{2}, P=0.016\right)$.

\section{Discussion}

Mechanical ocular trauma such as cataract surgery is known to be followed by a variety of ocular changes, 
which include miosis, conjunctival hyperaemia, breakdown of the blood-aqueous barrier and a rise in intraocular pressure. ${ }^{11}$ In 1965, Ambache et al ${ }^{12}$ discovered an unsaturated hydroxy acid, later known as PG, that produced miosis after iris stroking and paracentesis. There is strong evidence that PGs are at least partially responsible for the miosis observed following various forms of ocular trauma. Ocular tissues have the capacity to synthesise PGs. Furthermore, when exogenous PGs are administered topically or intracamerally, miosis occurs associated with ocular inflammation. PG synthesis inhibitors work by inhibiting the cyclo-oxygenase pathway preventing prostaglandin synthesis. In addition, some PG synthesis inhibitors have a relatively long duration of action and can offer other potential therapeutic benefits such as suppression of inflammation. Another mechanism of surgically induced miosis is a nerve-mediated pathway via substance P. ${ }^{11}$ Increased levels of substance $\mathrm{P}$ and calcitonin gene-related peptide, which is another potent miotic, have been found in diabetic humans and other mammalian species. ${ }^{13}$ The effect of PG synthesis inhibitors has not been well documented in diabetic patients.

PG synthesis inhibitors given topically or orally prior to cataract surgery have been shown to be effective in inhibiting surgically induced miosis. ${ }^{14-17}$ The use of adrenaline in the irrigating solution during cataract surgery has also been shown to maintain mydriasis. ${ }^{18,19}$ In our study, intraoperative miosis occurred more often in the diabetics. These results are similar to those reported by Zaczek and Zetterström, ${ }^{20}$ who studied surgically induced miosis in 32 diabetic patients. Our study differs from that of Zaczek and Zetterström ${ }^{20}$ because of the additional use of a topical PG synthesis inhibitor in our patients. As different statistical methods were used for analysis of the results in the study by Zaczek and Zetterström ${ }^{20}$ and our study, it is not possible to comment on the effect of diclofenac sodium in inhibiting surgically induced miosis in our diabetic patients. Although we did not record iris touch/prolapse during phacoemulsification, it is expected to have occurred at comparable frequency in the two groups.

Surgically induced miosis during cataract surgery is associated with a higher risk of complications such as iris trauma, uveitis, anterior capsule tears, posterior capsule rupture and zonular dehiscence leading to vitreous loss. ${ }^{21-23}$ A higher rate of capsular rupture reported in diabetics undergoing intracapsular ${ }^{24}$ or extracapsular ${ }^{25}$ extraction could be related to structural and chemical changes that are known to occur in the capsule in diabetes. The rate of conversion from phacoemulsification to extracapsular surgery is also increased in cases of surgically induced miosis. ${ }^{26}$ This is explained by the fact that a relatively small difference in pupillary diameter can make a significant difference in the surgical field. For example, it has been calculated that an increase in pupil by $0.8 \mathrm{~mm}$ results in a $26 \%$ increase in pupillary area, which has significant implications in surgical practice. ${ }^{11}$ These observations are important, especially in diabetics who are known to have a poorer outcome following cataract surgery as a result of increased incidence of fibrinous uveitis, cystoid macular oedema, worsening of diabetic retinopathy, and maculopathy. ${ }^{22,27}$

Previous studies have reported on the influence of the colour of the iris on the response of the pupil to various dilating agents. The colour of the iris depends on the amount of pigment in the melanocytes; dark irides contain more melanine. The race of a subject influences his response to dilating agents only to the extent that it determines the degree of pigmentation of his iris. Obianwu and $\mathrm{Rand}^{28}$ found that the mydriatic action of phenylephrine 3\% was inversely proportional to the degree of pigmentation of the iris in European, Indian, Chinese, and African students. The authors suggested that this difference was because of a more powerful reflex miosis in subjects with pigmented irides. Barbee and Smith $^{29}$ studied the mydriatic effect of phenylephrine, cyclopentolate, scopolamine, homatropine, and atropine in white subjects with blue and brown eyes, and in black subjects with dark eyes. Phenylephrine was found to be ineffective as a mydriatic in black individuals. Scopolamine, homatropine, and atropine yielded the greatest degree of mydriasis in the most uniform fashion in all three-eye-type groups. Increasing age can also result in miosis, which is most likely related to decreased sympathetic tone. ${ }^{30}$ To alleviate the effects of iris pigmentation and age in the results of our study, the diabetic patients were matched for race and age to healthy controls.

Our results indicate that the combination of phenylephrine $10 \%$ (sympathomimetic, active dilation) and cyclopentolate 1\% (anticholinergic, passive dilation) is a dilating regime equally effective in diabetics and healthy individuals. This is in accordance with previous studies. ${ }^{10,11,16,17,20,31}$ A previous report indicated that the pupils of diabetics who had previous laser treatment dilate less compared to those who did not require laser. ${ }^{10}$ In our study, only eight of the 76 patients had required laser treatment prior to cataract surgery (five had laser for maculopathy and three for proliferative retinopathy). The relatively small proportion of patients with severe diabetic retinopathy may be partially responsible for the comparable dilation of the whole group.

In our study, the duration of phacoemulsification was not significantly different between the diabetic and 
control group. The mean duration of phacoemulsification is comparable to that reported in other studies. ${ }^{31,32}$ Zaczek and Zetterström ${ }^{20}$ reported longer duration of phacoemulsification in diabetics compared to healthy controls. The authors attributed their results to the known anatomical changes in the anterior segment of the diabetic eye, the larger diabetic lens which is mainly a result of increased cortical thickening, ${ }^{6}$ and possible increased numbers of advanced cataracts in diabetics compared to their age-matched healthy controls. The area served by our Unit is that of the inner city of Birmingham, which is characterised by a high proportion of ethnic minorities. These groups of patients tend to present later with more advanced cataracts. This is reflected in the relatively large proportion of patients with white cataracts in both groups, especially in the control group in our study. It is possible that diabetic patients with cataract were referred to us earlier compared to their age-matched controls via the existing screening programme for diabetic retinopathy.

The results of our study suggest that surgically induced miosis occurs more often in diabetics. This finding together with the known limiting factors for favourable visual outcome following cataract surgery in the diabetics indicate that phacoemulsification in this group of patients should be performed by an experienced surgeon.

\section{Acknowledgements}

We thank the Nursing Staff in the Cataract Unit of the Birmingham and Midland Eye Centre for their help in recording the data read by the surgeon.

\section{References}

1 Kreines K, Rowe KW. Cataract and adult diabetes. Ohio Med J 1979; 75: 782-786.

2 Hiller R, Sperduto RD, Ederer F. Epidemiologic associations with cataract in the 1971-1972 National Health and Nutrition Examination Survey. Am J Ophthalmol 1983; 118: 239-248.

3 Podgor MJ, Cassel GH, Kannel WB. Lens changes and survival in a population-based study. N Engl J Med 1985; 313: 1438-1444.

4 Klein R, Moss SE, Klein BE et al. Relation of ocular and systemic factors to survival in diabetes. Arch Intern Med 1989; 149: 266-272.

5 Caird FI, Pirie A, Ramsell TG. Diabetes and the Eye. Oxford: Blackwell Scientific, 1969, p 131.

6 Bron AJ, Sparrow J, Brown NAP et al. The lens in diabetes. Eye 1993; 7: 260-275.

7 Smith SA, Smith SE. Evidence for a neuropathic aetiology in the small pupil of diabetes mellitus. Br J Ophthalmol 1983; 67: 89-93.
8 Hreidarsson AB. Pupil size in insulin-dependent diabetes: relationship to duration, metabolic control, and long-term manifestations. Diabetes 1982; 31: 442-448.

9 Fujii T, Ishikawa S, Uga S. Ultrastructure of the iris muscles in diabetes mellitus. Ophthalmologica 1977; 174: 228-239.

10 Huber MJE, Smith SA, Smith SE. Mydriatic drugs for diabetic patients. Br J Ophthalmol 1985; 69: 425-427.

11 Duffin RM, Camras CB, Gardner SK et al. Inhibitors of surgically induced miosis. Ophthalmology 1982; 89: 966-977.

12 Ambache N, Kavanagh L, Whiting J. Effect of mechanical stimulation on rabbits' eyes; release of active substance in anterior chamber perfusates. J Physiol (London) 1965; 176: 378-408.

13 Marfurt CF, Echtenkamp SF. The effect of diabetes on neuropeptide content in the rat cornea and iris. Invest Ophthalmol Vis Sci 1995; 36: 1100-1107.

14 Thaller VT, Kulshrestha MK, Bell K. The effect of preoperative topical flurbiprofen or diclofenac on pupil dilatation. Eye 2000; 14: 642-645.

15 Fry LL. Efficacy of diclofenac sodium solution in reducing discomfort after cataract surgery. J Cataract Refract Surg 1995; 21: 187-190.

16 Keulen-de Vos HCJ, Van Rij G, Renardel de Lavalette JCG et al. Effect of indomethacin in preventing surgically induced miosis. Br J Ophthalmol 1983; 67: 94-96.

17 Sachdev SM, Singh K, Talwar D et al. Comparative efficacy of diclofenac and flurbiprofen for maintaining pupillary dilatation during cataract surgery. Ophthalmic Surg 1994; 25: 92-94.

18 Gimbel HV. The effect of treatment with topical nonsteroidal anti-inflammatory drugs with and without intraoperative epinephrine on the maintenance of mydriasis during cataract surgery. Ophthalmology 1989; 96: 585-588.

19 Corbett MC, Richards AB. Intraocular adrenaline maintains mydriasis during cataract surgery. Br J Ophthalmol 1994; 78: 95-98.

20 Zaczek A, Zetterström C. Cataract surgery and pupil size in patients with diabetes mellitus. Acta Ophthalmol Scand 1997; 75: 429-432.

21 Krupsky S, Zalish M, Oliver M et al. Anterior segment complications in diabetic patients following extracapsular cataract extraction and posterior chamber intraocular lens implantation. Ophthalmic Surg 1991; 22: 526-530.

22 Cunliffe IA, Flanagan DW, George NDL et al. Extracapsular cataract surgery with lens implantation in diabetics with and without proliferative retinopathy. Br J Ophthalmol 1991; 75: 9-12.

23 Guzek JP, Holm M, Cotter JB et al. Risk factors for intraoperative complications in 1000 extracapsular cataract cases. Ophthalmology 1987; 94: 461-466.

24 Caird RI, Hutchinson M, Pirie A. Cataract and diabetes. $\mathrm{Br}$ Med J 1964; 2: 665-668.

25 Kuchle M, Schonherr U, Dieckmann U. Risk factors for capsular rupture and vitreous loss in extracapsular cataract extraction. The Erlangen Ophthalmology group. Fortschr Ophtalmol 1989; 86: 417-421.

26 Dada T, Sharma N, Vajpayee RB et al. Conversion from phacoemulsification to extracapsular cataract extraction: incidence, risk factors and visual outcome. J Cataract Refract Surg 1998; 24: 1521-1524.

27 Pollack A, Leiba H, Bukelman A et al. Cystoid macular oedema following cataract extraction in patients with diabetes. Br J Ophthalmol 1992; 76: 221-224. 
28 Obianwu HO, Rand MJ. The relationship between the mydriatic action of ephedrine and the colour of the iris. Br J Ophthalmol 1965; 49: 264-270.

29 Barbee RF, Smith WO. A comparative study of mydriatics and cycloplegic agents. Am J Ophthalmol 1957; 44: 617-622.

30 Moses RA, Hart WM Jr (eds). Adler's Physiology of the Eye. Clinical Application, 9th edn. Mosby Year Book: St Louis, MO, 1992, p. 414.
31 Solomon KD, Turkalj JW, Whiteside SB et al. Topical 0.5\% ketorolac vs $0.03 \%$ flurbiprofen for inhibition of miosis during cataract surgery. Arch Ophthalmol 1997; 115: 1119-1122.

32 Chakrabarti A, Singh S, Krishnadas R. Phacoemulsification in eyes with white cataract. J Cataract Refract Surg 2000; 26 1041-1047. 